\title{
Neural stem cells preferentially migrate to glioma stem cells and reduce their stemness phenotypes
}

\author{
SUOJUN ZHANG ${ }^{*}$, RUIFAN XIE* , TIANYUAN ZHAO, XIONG YANG, \\ LIN HAN, FEI YE, TING LEI and FENG WAN
}

\begin{abstract}
Department of Neurosurgery and Chinese-German Lab of Molecular Neurooncology, Tongji Hospital, Tongji Medical College, Huazhong University of Science and Technology, Wuhan 430030, P.R. China
\end{abstract}

Received June 28, 2014; Accepted August 12, 2014

DOI: $10.3892 /$ ijo.2014.2629

\begin{abstract}
Glioma stem cells (GSCs), characterized by selfrenewal, multi-potentiality and tumorigenicity, are responsible for the tumor propagation, recurrence and resistance to traditional treatments, representing a critical therapeutic target. Neural stem cells (NSCs) possess inherent tropism to brain tumor cells and inhibit their growth. However, there is a limited understanding of the mechanism underlying NSC tropism and the effect of NSC migration on GSC stemness phenotypes. In the present study, we showed that GSCs exhibited enhanced chemotax is for NSC tropism compared with their differentiated cells. Chemokines secreted by GSCs contributed to the targeted migration of NSCs. Hypoxia enhanced NSC tropism via the upregulated chemokine expression of GSCs, such as VEGF, EGF and bFGF. In vitro migration of NSCs induced GSC differentiation and reduced stem-like phenotypes. Moreover, in vivo data provided direct evidence that transplanted NSCs could migrate to GSCs from either the homolateral or contralateral brain injection site, which prolonged the survival of grafted mice. Taken together, these findings show that NSCs preferentially migrate to GSCs and reduce their stemness phenotypes, raising the intriguing possibility that the targeted migration of NSCs can be applied as a novel therapeutic strategy to target these intractable brain tumors.
\end{abstract}

\section{Introduction}

Glioblastoma (GBM) is the most frequent and malignant brain tumor in the human central nervous system (CNS). Despite

Correspondence to: Dr Feng Wan, Department of Neurosurgery and Chinese-German Lab of Molecular Neurooncology, Tongji Hospital, Tongji Medical College, Huazhong University of Science and Technology, 1095 Jiefang Avenue, Wuhan 430030, P.R. China E-mail:wanruiyan@hotmail.com

E-mail: tjhneurosurgery@163.com

*Contributed equally

Key words: neural stem cells, glioma stem cells, glioblastoma, migration research progress on its molecular mechanisms and optimal treatments, including aggressive surgery, chemotherapy, radiotherapy and immunotherapy, the prognosis for patients with GBM is still relatively poor $(1,2)$. The current model of glioma stem cells (GSCs) has significantly contributed to a better understanding of the cellular origin and failure of current conventional treatments $(3,4)$. GSCs, which are characterized by their ability to self-renew, multi-potentiality and tumorigenicity, are thought to contribute to the tumor propagation, recurrence and resistance to chemotherapy and radiotherapy, and they represent a critical therapeutic target (5-9).

Neural stem cell/neural progenitor cell (NSC/NPC) transplantation was initially applied in the study of Parkinson's disease $(10,11)$ and subsequently in spinal cord injury (12), stroke and multiple sclerosis $(13,14)$. Until 2000, several research groups demonstrated that NSCs exhibited a unique migratory capacity of efficiently crossing the blood-brain barrier to target brain tumors located distantly from the original transplant site throughout the experimental brain (15-17). Subsequent studies have proposed that NSCs may possess some natural abilities to suppress tumor growth and to induce tumor cell apoptosis. For example, Staflin and colleagues injected rat embryonic neural progenitor cell lines into the nucleus caudatus of Fisher rats combined with glioma cells and found that injected NPCs could inhibit glioma outgrowth in vivo, resulting in an extension in the life span of glioma inoculated rats (18). Consistent with this finding, Glass et al showed that endogenous NPCs in the adult brain exhibited a strong tropism for glioblastomas (19). The addition of neural precursors into glioblastoma grafts promoted tumor cell death and prolonged survival in vivo. Furthermore, the NSC inherent tropism towards brain tumors resulted in the pursuit of applying NSCs as a promising therapeutic tool and/or vehicle for tracking and suppressing malignant glioma cells (20-25). However, limitations exist when applying a small number of NSCs to restrain the overwhelming number of bulk tumor cells. In the present study, we aimed at exploring the preferential tropism of NSCs to GSCs and the effect of migrated NSCs on GSC stemness phenotypes.

\section{Materials and methods}

Cell culture. Human neural stem cells (NSCs; ReNcell CX immortalized cells, SCC007, Millipore, MA, USA) were 
cultured as an adherent monolayer in a laminin-coated flask in NSC maintenance medium (Millipore) supplemented with epidermal growth factor (EGF; PeproTech Inc., NJ, USA) and basic fibroblast growth factor (bFGF; PeproTech) at $20 \mathrm{ng} / \mathrm{ml}$, respectively, and were used within ten passages as recommended. Human primary glioma stem cells (GSCs) were derived from tumor resection. Tumor tissue was enzymatically dissociated and cultured as floating neurospheres in serum-free supplemented medium [DMEM/F-12 medium containing 20\% BIT serum-free supplement (Stemcell Technologies Inc., Vancouver, Canada), EGF and bFGF at $20 \mathrm{ng} / \mathrm{ml}$, respectively] (26,27). Glioma U251 stem cells (U251-SC) were also cultured as non-adherent neurospheres in serum-free supplemented medium as previously described, which were derived from the human glioma cell line U251. GSCs of more than six passages were used for further experiments. To induce GSC differentiation, we used the conventional serum-containing medium (10\% FBS in DMEM) for seven days. For hypoxic incubation, the cells were grown at $1 \%$ oxygen for $24 \mathrm{~h}$ in a hypoxic workstation. The Human human fibroblast cell line HFL1 (ATCC ${ }^{\circledR}$ CCL-153 ${ }^{\mathrm{TM}}$ ) was used as control cells.

Immunofluorescence staining. The primary antibodies used in this study included rabbit anti-human CD133 (1:500, Abcam, MA, USA) and mouse anti-human Nestin (1:500, Abcam) and incubated for $16 \mathrm{~h}$ at $4{ }^{\circ} \mathrm{C}$, followed by detection with the corresponding fluorescent secondary antibodies. Nuclei were stained with DAPI, and the slides were detected using fluorescence microscopy.

CD133 flow cytometry analysis. Up to $10^{6}$ cells were resuspended in the recommended buffer (containing PBS pH 7.2, $0.5 \% \mathrm{BSA}$, and $2 \mathrm{mM}$ EDTA) and incubated for $10 \mathrm{~min}$ at $4^{\circ} \mathrm{C}$ with CD133/1 (AC133) antibody (1:11; Miltenyi Biotec, Bergisch Gladbach, Germany). Mouse IgG1 (1:11; Miltenyi Biotec) was used as isotype control antibody. CD133 detection and analysis were performed on BD FACS Aria.

Western blot analysis. The primary antibodies used included rabbit anti-human CD133 (1:500, Abcam), mouse anti-human Nestin (1:500, Abcam), rabbit anti-human GFAP (1:500, Abcam) and mouse anti-human GAPDH (1:1000, Boster, Wuhan, China).

Conditioned medium (CM). GSC and U251-SC conditioned medium were harvested as previously described (27). For hypoxic cells CM, GSCs and their differentiated cells were shifted to DMEM/F-12 medium without any supplement at $1 \%$ oxygen for $24 \mathrm{~h}$. The $\mathrm{CM}$ was harvested with viable cell counting and stored at $-80^{\circ} \mathrm{C}$ for further use. To obtain the growth factor medium, VEGF, EGF and bFGF were added into DMEM/F12 at a concentration of $20,10,5$ and $0 \mathrm{ng} / \mathrm{ml}$ immediately before use.

Cell migration transwell assay. The in vitro migration of NSCs to GSCs or differentiated cells was detected using Transwell assay. Each well of 24-well cell culture plates was separated into two chambers by an insert membrane of $8-\mu \mathrm{m}$ pores. Briefly, $600 \mu \mathrm{l}$ of CM was placed into each lower chamber and DMEM/F12 without supplement was used as a basal migra- tion control. NSCs $\left(1 \times 10^{4}\right.$ cells in $100 \mu 1$ of DMEM/F12) were then seeded into the upper chamber. After $6 \mathrm{~h}$ of incubation at $37^{\circ} \mathrm{C}$, NSCs in the chambers were fixed with $95 \%$ ethanol. Non-migrating cells on the upper side of the insert membrane were wiped off. Migrating cells on the bottom of the membrane were stained using $0.5 \%$ crystal violet and quantified under a microscope.

Mixed culture of NSCs and GSCs. For mixed culture, NSCs and GSCs were labeled using the fluorescent tracer Dio and Dil (Molecular Probes, Inc., Eugene, OR, USA), respectively, as recommended. Single cell suspensions of NSCs, GSCs or $\mathrm{U} 251-\mathrm{SCs}$ were prepared at a density of $1 \times 10^{6} / \mathrm{ml}$ in serum-free culture medium and $5 \mu \mathrm{l}$ of the cell-labeling solution (Dio for NSC, Dil for GSC and U251-SC) was added per ml of cell suspension. After incubation for $20 \mathrm{~min}$ at $37^{\circ} \mathrm{C}$ and a rinse in warm $\left(37^{\circ} \mathrm{C}\right)$ medium, $2 \times 10^{5}$ NSCs were seeded in per laminin-coated 6 -well plates as an adherent monolayer and $1 \times 10^{5}$ GSCs were seeded in per uncoated plates as suspended neurospheres. On the second day, the GSC neurospheres were mixed and cultured with NSCs. The migration of NSCs and proliferation of GSC neurospheres were detected using fluorescent microscopy.

Co-culture of NSCs and GSCs. For co-culture of NSCs and GSCs, the transwell assay was used. Each well of 6-well cell culture plates was separated into two chambers by an insert membrane of $0.4-\mu \mathrm{m}$ pores. Briefly, $2 \times 10^{5} \mathrm{NSCs}$ (or HFL1 cells as control) were cultured as an adherent monolayer in the laminin-coated lower chamber and $1 \times 10^{5}$ GSCs were cultured as neurospheres in the upper chamber. NSC maintenance medium supplemented with EGF and bFGF at $2 \mathrm{ng} / \mathrm{ml}$, respectively, was used and added every three days for one week. The expression of cell markers and the self-renewal ability of GSCs co-cultured with NSCs (or control cells) were subsequently determined.

Secondary neurosphere formation assay. For secondary neurosphere formation assay, primary GSC neurospheres co-cultured with NSCs (or control cells) after a week were harvested and dissociated mechanically using a mechanical cellular filter. Single cell suspension washed with PBS was confirmed microscopically and suspended at 5,000 cells $/ \mathrm{ml}$ in serum-free supplemented medium. Using the limiting dilution assay, 1,000, 500, 200, 100, 50, 20 and 10 cells in $200-\mu 1$ suspensions were plated into 12 wells of each row in a 96-well microplate. Fifty $\mu 1$ of serum-free supplemented medium was added to each well every third day for two weeks. Neurospheres (non-adherent, tight and spherical masses $>75 \mu \mathrm{m}$ in diameter) per well were quantified using an ocular micrometer. To confirm the gradient dilution results described above, we performed a more stringent clonal assay by plating single cells into a 96-well plate, i.e., one viable cell per well, and at the end of two weeks, the wells containing the clonal spheres derived from a single cell were calculated.

Intracranial cell migration and tumorigenesis assay. Female BALB/c nude mice, six to eight weeks of age, were housed under specific pathogen-free conditions. All animal experimental protocols were approved by the Institutional Animal 

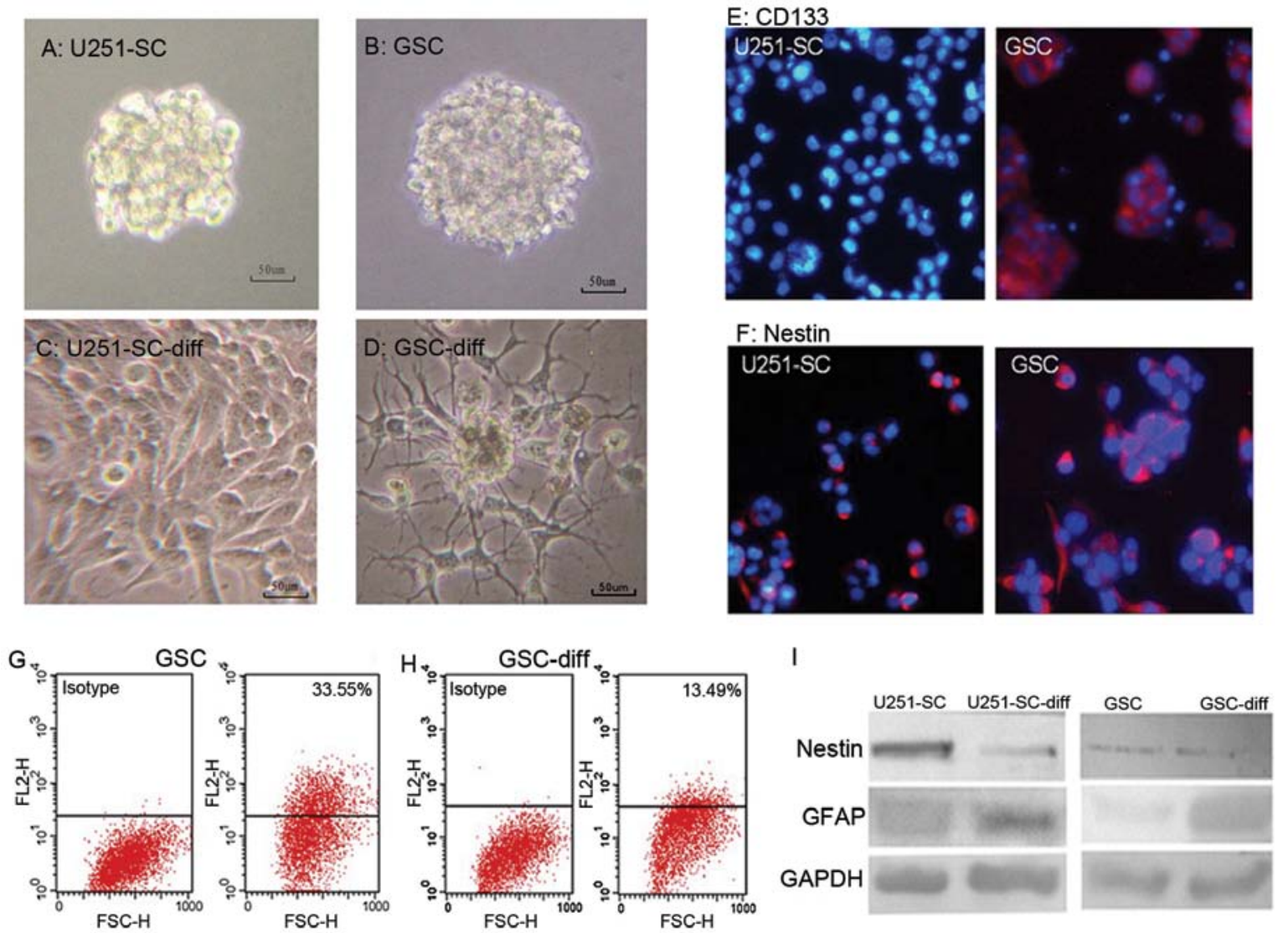

।

Figure 1. Establishment of GSCs and their differentiated cells. (A and B) Primary GSCs and U251-SC displayed a growth pattern with floating neurospheres in uncoated flask and serum-free supplemented medium, (C and D) while their differentiated cells (GSC-diff and U251-SC-diff) attached to the cultivation flask and branched out. (E and F) Immunofluorescence staining showed high expression of stem cell markers CD133 and Nestin in GSCs, except no detection of CD133 in U251-SC. (G and H) Flow cytometry analysis showed an enhanced CD133 expression in primary GSCs compared with GSC-diff and (I) increased Nestin expression while decreased GFAP expression were further confirmed by western blot assay.

Care and Use Committee, Huazhong University of Science and Technology. Briefly, NSCs and GSCs were labeled using the fluorescent tracer Dio (green fluorescent) and Dil (red fluorescent), respectively, as previously described. For the group of unilateral intracranial xenografts, GSCs $\left(1 \times 10^{5}\right.$ cells in $5 \mu \mathrm{l} \mathrm{PBS}$ ) were stereotactically implanted into the right basal ganglia of the nude mouse brain (AP $+1.0 \mathrm{~mm}, \mathrm{ML}$ $+2.0 \mathrm{~mm}$ and DV $-3.0 \mathrm{~mm}$ from bregma and dura) using a 10- $\mu$ l Hamilton syringe at a speed of $1 \mu \mathrm{l} / \mathrm{min}$ and the NSCs ( $2 \times 10^{5}$ cells in $5 \mu \mathrm{l}$ PBS) were then implanted $2.0 \mathrm{~mm}$ to the right of the injection site (which was AP $+1.0 \mathrm{~mm}, \mathrm{ML}$ $+4.0 \mathrm{~mm}$ and DV $-3.0 \mathrm{~mm}$ from bregma and dura). For the bilateral intracranial xenograft group, GSCs were implanted at the same site, and the NSCs were symmetrically injected into the contralateral side of the mouse brain, $2.0 \mathrm{~mm}$ left from bregma. Injection of HFL1 cells instead of NSCs served as the control. To detect the migration of NSCs, mouse brain samples were collected at one and two weeks and were snap-frozen for subsequent examination. To determine tumorigenicity, the mice were maintained until weight loss of $>10 \%$ occurred or when neurological signs appeared.

Statistical analyses. Statistics were performed using SPSS 17.0 software. Comparisons among the groups were performed with analysis of variance (ANOVA) or Student's t-test. Significance was established at $\mathrm{p}<0.05$.

\section{Results}

Compared with differentiated cells, GSCs induced enhanced NSC tropism and secreted more chemotactic factors. To determinate the migratory capacity of NSCs to GSCs and their differentiated cells, we first established human GSCs from primary glioblastoma and U251 glioma stem cells (U251-SC) from the U251 glioma cell line. GSCs were cultured as floating neurospheres in an uncoated flask and serum-free supplemented medium (Fig. 1A and B). The GSCs and U251-SC were derived from serum-containing medium in seven days. Upon differentiation in serum-containing medium, the GSC neurospheres quickly attached to the culture flask and branched out, instead of forming a suspension (Fig. 1C and D). Cell markers (CD133, Nestin and GFAP) of GSCs and their differentiated cells showed contrasting effects. A marked decrease in Nestin and/or CD133 expression in GSCs and a notable increase in GFAP expression in differentiated cells were revealed (Fig. 1E-I).

To detect NSC migration in vitro, conditioned medium of GSCs and their differentiated cells were harvested. An in vitro migration assay was used to test the migratory capacity of NSCs to GSCs and their differentiated cells. These results showed that the conditioned medium of GSCs and their differentiated cells caused significantly more NSCs to migrate from the top well, through the porous membrane to the lower surface when 


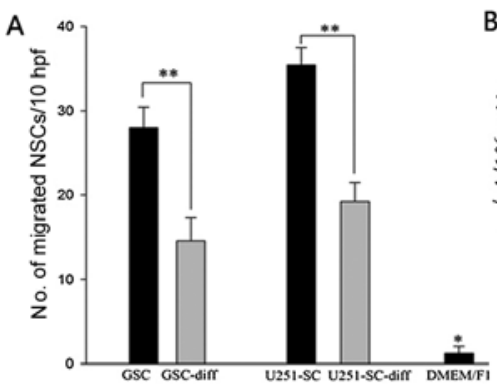

B
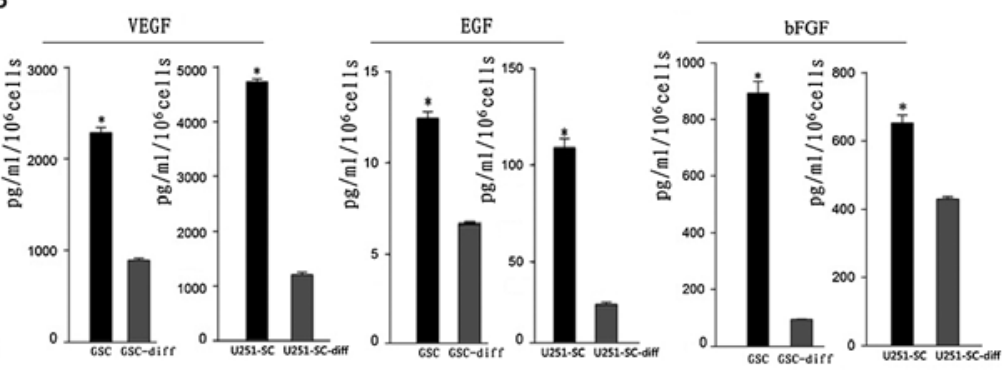

C
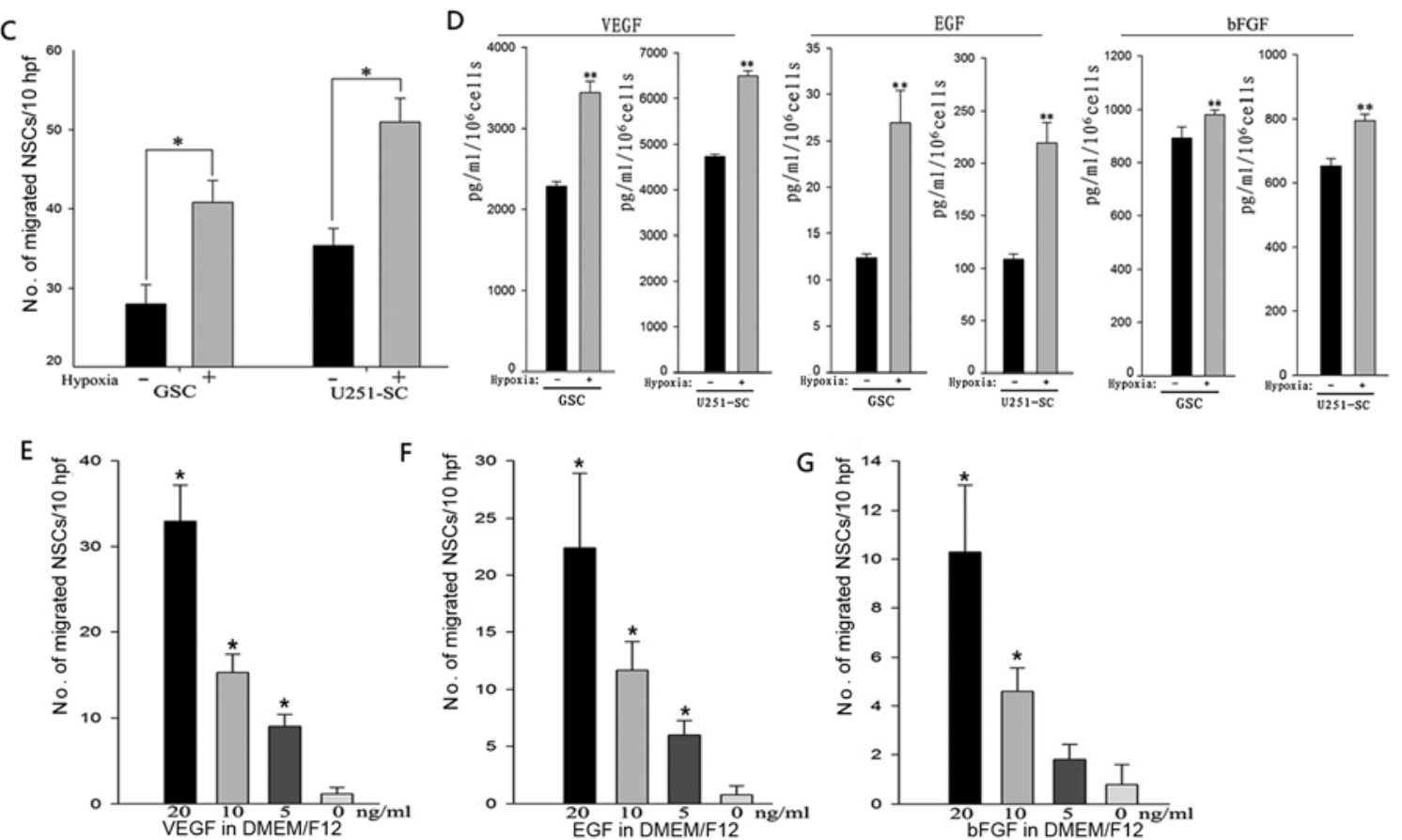

Figure 2. Evaluation of the in vitro migrating capability of NSCs and the regulating factors. (A) Conditioned medium of GSCs and their differentiated cells caused significantly enhanced NSC tropism, compared to unconditioned medium ( $\left.{ }^{*} \mathrm{P}<0.001\right)$. Notably, NSCs preferentially migrated toward conditioned medium of GSCs, compared with that of their differentiated cells $\left({ }^{* *} \mathrm{P}<0.05\right)$. (B) An incremental secretion of chemotactic factors (VEGF, EGF and bFGF) was detected in the conditioned medium of GSCs by ELISA assay, compared with counterpart of heir differentiated cells ("P<0.05). (C) Hypoxia promoted NSC tropism to GSCs and (D) upregulated expressions of chemotactic factors (VEGF, EGF and bFGF) were detected ( $(*$ P $<0.05)$. (E-G) A concentration-dependent migration of NSCs was elicited by VEGF, EGF and bFGF ( $\mathrm{P}<0.05)$.

compared to unconditioned medium ( $\mathrm{P}<0.001)$. Interestingly, NSCs exhibited robust mobility toward conditioned medium of GSCs, compared to that of their differentiated cells, with an increase of $\sim 2$-fold in primary GSCs and U251-SCs (Fig. 2A; $\left.{ }^{* *} \mathrm{P}<0.05\right)$. Next, we detected the levels of chemotactic factors (VEGF, EGF and bFGF) in the conditioned medium. Notably, an incremental secretion of chemotactic factors was detected in the conditioned medium of GSCs compared to their differentiated cells (Fig. 2B; ${ }^{*} \mathrm{P}<0.05$ ).

Hypoxia promoted NSC tropism to GSCs by upregulating the expression of chemotactic factors, which played critical roles in NSC migration. Hypoxia has been identified to play a critical role in promoting tropism and mobilization of multiple stem cells, including NSCs. To assess the effects of hypoxia on NSC migration toward GSCs, we preconditioned GSCs to DMEM/F-12 medium without any supplement at $1 \%$ oxygen for $24 \mathrm{~h}$ and then harvested the hypoxic preconditioned medium for in vitro migration assay. Increasing NSC migration rates of $\sim 1.5$ times were found in primary GSCs and U251-SC (Fig. 2C; ${ }^{*} \mathrm{P}<0.05$ ).
Since hypoxia promoted NSC tropism to GSCs, we further detected the effects of hypoxia on the expression of chemotactic factors. Quantified GSCs were preconditioned in the DMEM/F-12 medium without any supplement at $1 \%$ oxygen for $24 \mathrm{~h}$ and the hypoxic preconditioned medium were then harvested for ELISA analysis. These results showed that primary GSCs and U251-SC under hypoxia secreted much greater amounts of VEGF, EGF and bFGF compared to cells cultured in normoxia (Fig. 2D; ${ }^{* *} \mathrm{p}<0.05$ ).

To confirm the role of chemokines in NSC migration, we investigated the effects of grow factor concentration gradient medium on NSC tropism. VEGF, EGF and bFGF were added into DMEM/F12 at a concentration gradient of 20, 10, 5 and $0 \mathrm{ng} / \mathrm{ml}$ for in vitro migration assay. A concentrationdependent migration of NSCs was elicited by VEGF, EGF and bFGF, which suggested that chemotactic factors induced by hypoxia played critical roles in NSC migration (Fig. 2E-G).

In vitro migration of NSCs to GSCs displays cytostatic effect. To explore the effects of in vitro NSC migration on GSC growth, we labeled NSCs and GSCs using the fluores- 

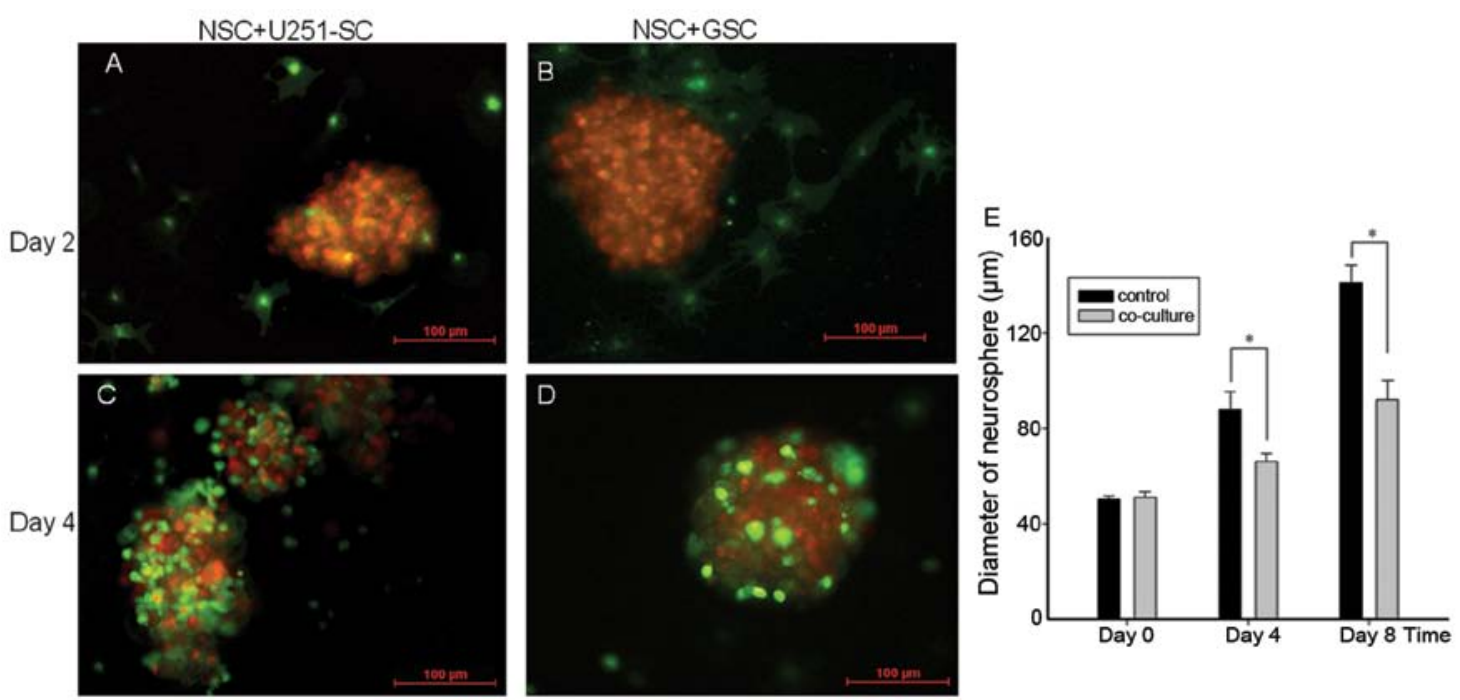

Figure 3. Assessment of the effect of in vitro NSC migration on GSC growth. (A and B) Co-cultured NSCs (labeled with Dio, green) with GSCs (labeled with Dil, red) on the second day. (C and D) On the forth day after co-culture, numerous NSCs were detected surrounding the whole GSC neurospheres. (E) Compared with the control, co-culture with NSCs caused an inhibition of GSC neurosphere growth, with remarkable decrease in neurosphere diameters $($ ( $\mathrm{P}<0.05)$.
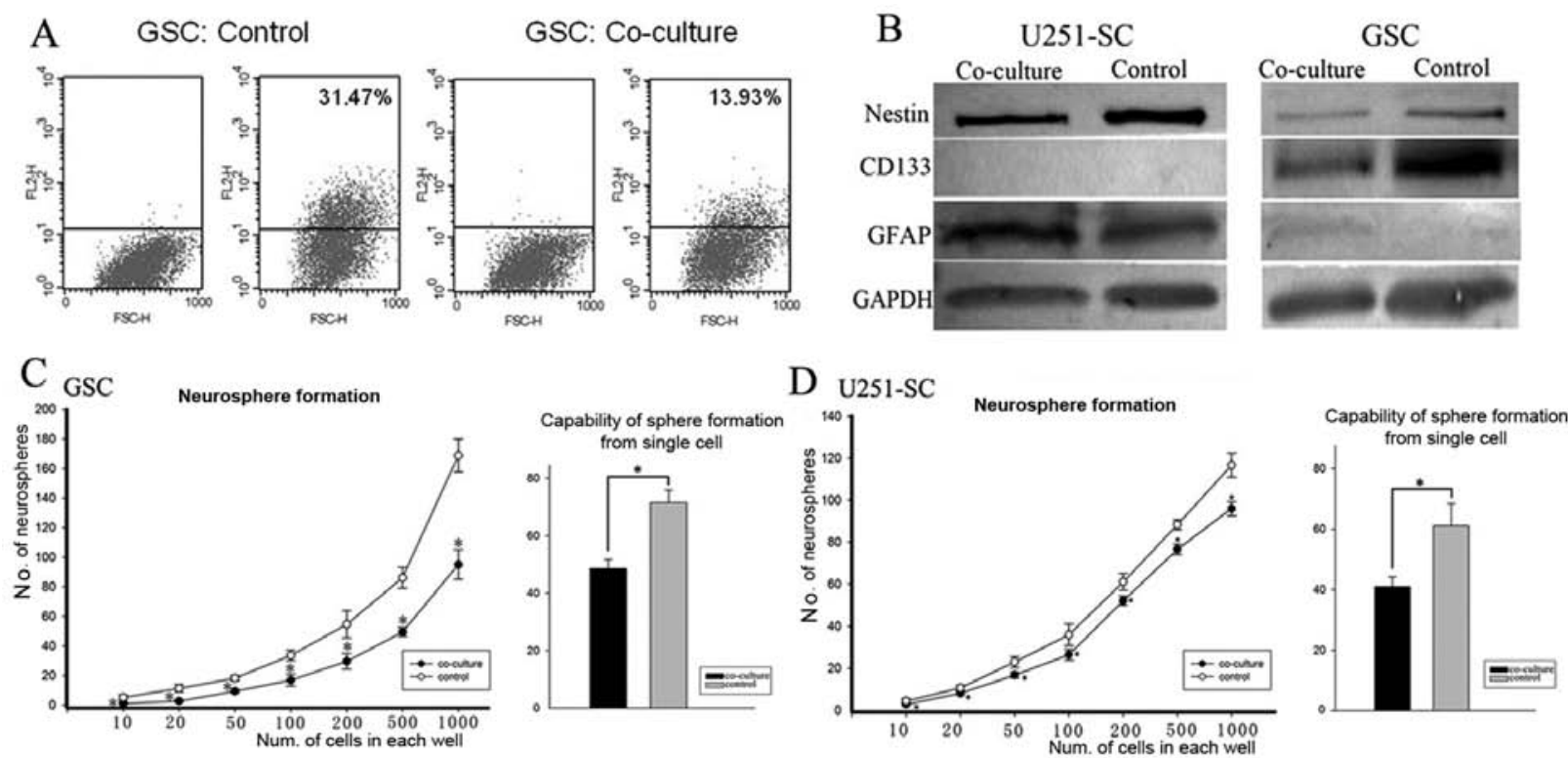

Figure 4. Effect of co-culture with NSCs on GSC stemness phenotypes. (A) Flow cytometry analysis showed co-culture with NSCs led to an decreased CD133 expression in GSCs. (B) Reduced stem cell marker expressions in GSCs after co-culture with NSCs were further confirmed by western blot assay, with decreased expression of CD133 and Nestin while increased GFAP expression. (C and D) Limiting dilution clone assay and single-cell sphere formation assay showed neurosphere formation capability of GSCs was reduced through co-culture with NSCs ("P<0.05).

cent tracer Dio (green) and Dil (red), respectively, and we co-cultured labeled NSCs and GSC neurospheres on the second day. On the second day after the co-culture, NSCs were detected to migrate toward GSC neurospheres (Fig. 3A and B). On the fourth day after the co-culture, numerous NSCs had migrated to the GSC neurospheres and surrounded the entire neurospheres (Fig. 3C and D).

To assess the effects of NSC migration on GSC neurosphere growth, the diameters of GSC neurospheres were measured and analyzed using a computerized fluorescence microscope after co-culture. Compared with the control, co-cultures with NSCs caused an inhibition of GSC neurosphere growth, with a remarkable decrease in the neurosphere diameters (Fig. 3E; $\mathrm{P}<0.05$ ). Stem-like phenotypes and self renewal capability of GSCs were reduced after co-culture with NSCs. For the purpose of detecting the effects of NSC migration on GSC stemness, we co-cultured GSCs with NSCs. After one-week of co-culture, CD133-positive cells were analyzed using the flow cytometry assay. Compared with the control (co-culture with HFL1), there was a notable decrease in the rate of CD133-positive GSC cells after co-culture with NSCs, which indicated a decrease in the GSC subpopulation (Fig. 4A). Further detection using western blot analyses confirmed this finding. The expression of CD133 and Nestin in GSCs 


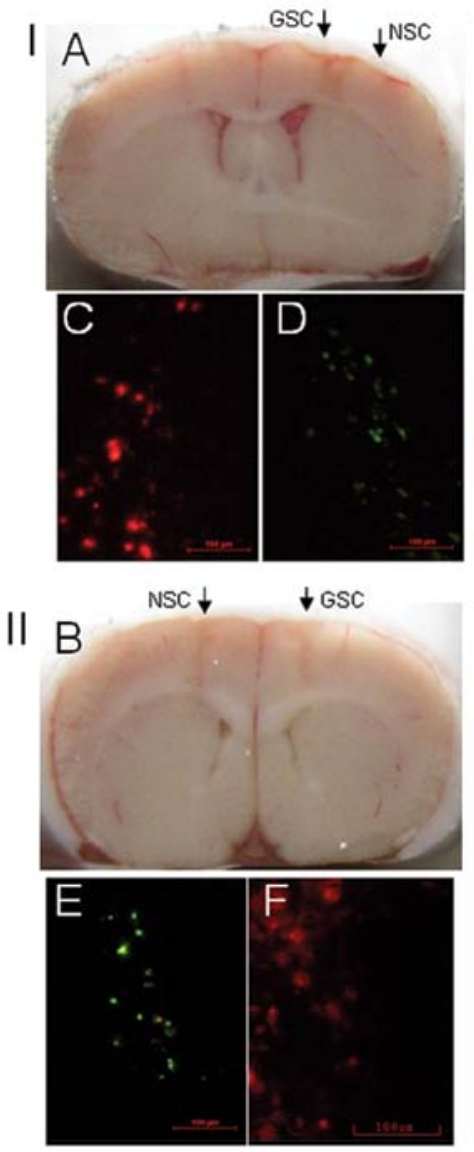

Week 1 st
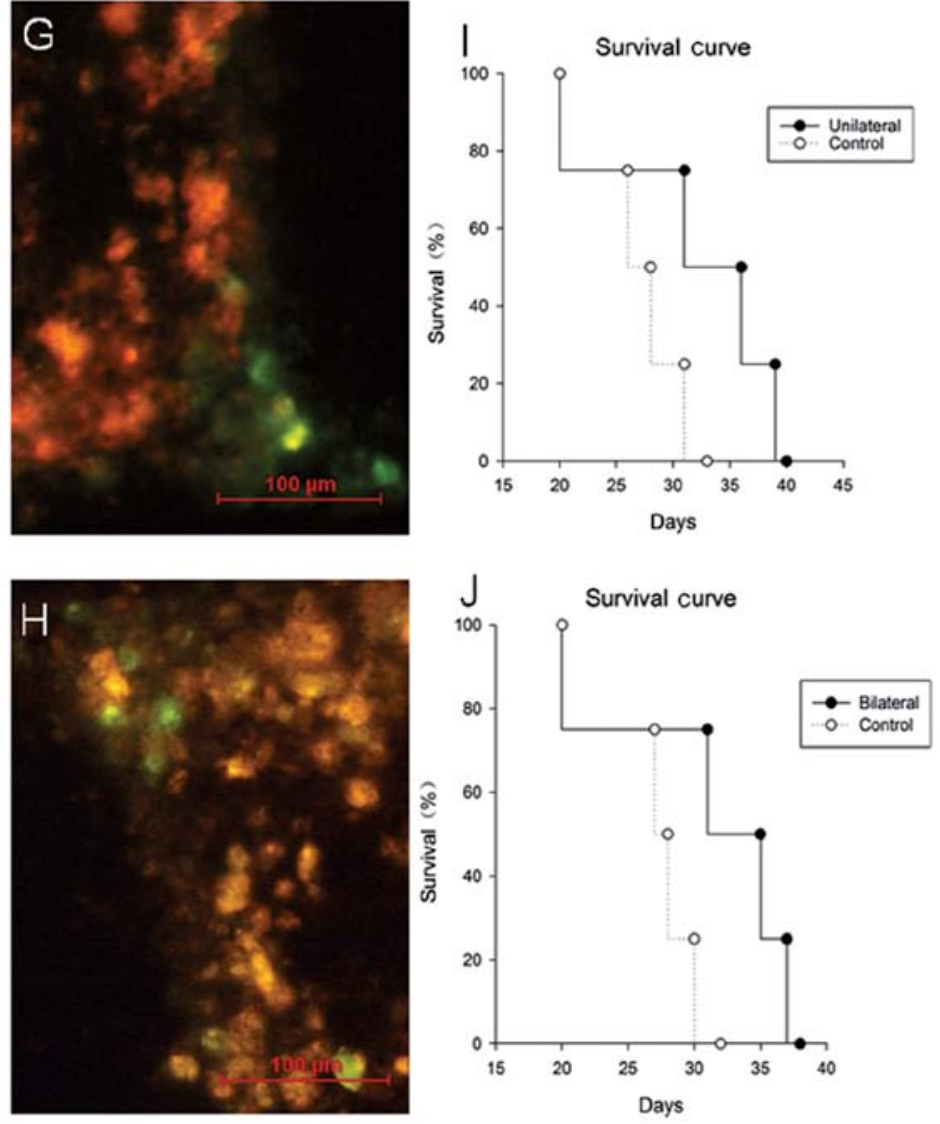

Figure 5. In vivo migration of NSCs to GSCs improved survival. NSCs (labeled with Dio, green) and GSCs (labeled with Dil, red) were implanted stererotactically as unilateral (I.A) or bilateral (II.B) implant. (C and F) Implanted region of GSCs with no migrated NSCs from implanted region of NSCs (D and E) at the end of the first week after the xenografts. (G and H) Migrated NSCs around GSCs were detected in both unilateral and bilateral grafted mouse brains at the end of the second week. (I and J) Prolonged survivals in both unilateral and bilateral grafted mice, compared to the control group.

were decreased, while GFAP expression was increased in the co-culture (Fig. 4B). The data indicated that GSCs were induced to differentiate in co-cultures with NSCs.

The ability to self-renew is one of the most important stem cell phenotypes. The capability to neurosphere formation in vitro has been applied to identify self-renewal of GSCs and is considered a significant and independent predictor of the clinical outcome of glioma patients (28). As manifested by the limiting dilution clone assay and further supported by the more stringent single-cell sphere formation assay, GSCs after co-culture showed decreased self-renewal potentiality (Fig. 4C and D; $\mathrm{P}<0.05$ ).

In vivo migration of NSCs to GSCs improves survival. To detect the in vivo migration of NSCs to GSCs, we labeled NSCs with Dio (green fluorescent) and GSCs with Dil (red fluorescent), respectively. Next, GSCs and NSCs were implanted stereotactically either unilaterally (Fig. 5A) or bilaterally (Fig. 5B) in the mouse brain. At the end of the first week after the xenograft, there was no migrating NSCs detected nearby the implanted region of GSCs (Fig. 5C and F). After two weeks, the migrated NSCs around the GSCs were detected in both the unilateral and bilateral grafted mouse brains (Fig. 5G and $\mathrm{H}$ ).

To determine the effect of NSC migration on GSC tumorigenicity, tumor-burdened mice that developed weight loss $>10 \%$ or showed the presence of neurological signs were recorded. There were prolonged survivals in both the unilateral and bilateral grafted mice, compared to the control group (Fig. 5I and J), suggesting that the in vivo migration of NSCs to GSCs improved survival.

\section{Discussion}

Failure of current therapies for malignant gliomas suggests that effective treatment may be dependent on the development of new therapeutic strategies, which can eradicate the arch criminal origin and/or the residual reservoirs of tumor cells left behind after conventional treatments. Increasing studies on GSCs have provided support for a new paradigm in tumor biology and therapeutic targeting. It has been identified that NSCs exhibit an inherent tropism to target malignant gliomas and inhibit tumor cell growth (15-19). In the present study, we evaluated the specific tropism of NSC to GSCs and its potential therapeutic significance.

There have been numerous studies demonstrating that stem cell migration is largely driven by various chemotactic cytokines (29-31). Tumor upregulated expression of chemotactic cytokines and the microvasculature contain relevant guidance signals for NSC tropism toward malignant brain tumors $(32,33)$. We have previously demonstrated that the secretion of 
VEGF and bFGF by GSCs was dramatically higher than that of differentiated tumor cells (27). Furthermore, considering that EGF, in addition to bFGF, is one of the key growth factors, which suppresses differentiation and enables the in vitro expansion of highly pure populations of stem cells (34-36), we further compared the distinct secretion of EGF by GSCs and their differentiated cells, which showed a similarly high secretion by GSCs. A concentration-dependent NSC migration elicited by these factors confirmed their critical roles in NSC tropism. These findings strongly suggested that GSCs might possess enhanced tropism for NSC compared with their differentiated counterparts.

Furthermore, we found that this growth factor-regulating NSC migration was dramatically upregulated by hypoxia, which was consistent with previous reports that hypoxia plays an important role in regulating NSC migration toward gliomas $(29,37,38)$. Importantly, given the intratumoral hypoxic gradient in the tumor microenvironment, which may drive heterogeneous GSC distribution (39), NSCs could preferentially target the GSC compartment in the tumor mass. Indeed, NSCs exhibited a robust mobility toward GSCs in vitro in a migration model compared to their differentiated cells. Importantly, our results showed that the preferential migration of NSCs to GSCs exhibited an antitumor effect. In vitro, a mixed culture of NSCs with GSCs resulted in a direct inhibition of GSC neurosphere growth. Such antitumor effects have been previously reported for both endogenous neural precursor cells (19) and exogenously added NSCs from newborn mice $(16,18)$. Interestingly, our results demonstrated that co-culture with NSCs induced GSC differentiation, attenuated stem cell marker expression and reduced their self-renewal capability. Importantly, in vivo, the orthotopic xenografted NSCs prolonged the survival of the mice bearing xenografted GSCs. Consistently, it has been demonstrated that neural precursor cells may suppress the tumorigenicity of GSCs by releasing bone morphogenetic protein-7 (40). Taken together, NSCs exhibited preferential tropism to GSCs and reduced their stemness phenotypes.

Intracranially or intravenously injected genetically engineered NSCs have been applied to eradicate the invasive tumor microsatellite and/or remnant tumor cells prior to their development into recurrent gliomas (17,41-43). Under the scenario of the GSCs model, our results introduce the potential strategy of applying exogenous NSCs to specifically target and reduce the most invasive and therapy-resistant tumor stem cell compartment, which can eventually result in tumor relapse. Thus, it would be interesting to further explore when and how the endogenous NSCs undergo this process and what could be the ultimate fate of the migrated NSCs when encountering their aberrant counterparts.

\section{Acknowledgements}

This study was supported by grants of the Natural Science Foundation of China (NSFC: 81272423,81101620 and 8142058).

\section{References}

1. Wen PY and Kesari S: Malignant gliomas in adults. N Engl J Med 359: 492-507, 2008.
2. Fuller GN and Scheithauer BW: The 2007 Revised World Health Organization (WHO) Classification of Tumours of the Central Nervous System: newly codified entities. Brain Pathol 17: 304-307, 2007

3. Galli R, Binda E, Orfanelli U, et al: Isolation and characterization of tumorigenic, stem-like neural precursors from human glioblastoma. Cancer Res 64: 7011-7021, 2004.

4. Singh SK, Clarke ID, Terasaki M, et al: Identification of a cancer stem cell in human brain tumors. Cancer Res 63: 5821-5828, 2003.

5. Bao S, Wu Q, McLendon RE, et al: Glioma stem cells promote radioresistance by preferential activation of the DNA damage response. Nature 444: 756-760, 2006.

6. Sakariassen PO, Immervoll $\mathrm{H}$ and Chekenya M: Cancer stem cells as mediators of treatment resistance in brain tumors: status and controversies. Neoplasia 9: 882-892, 2007.

7. Eyler CE and Rich JN: Survival of the fittest: cancer stem cells in therapeutic resistance and angiogenesis. J Clin Oncol 26: 2839-2845, 2008

8. Clarke MF, Dick JE, Dirks PB, et al: Cancer stem cells - perspectives on current status and future directions: AACR Workshop on cancer stem cells. Cancer Res 66: 9339-9344, 2006.

9. Liu Q, Nguyen DH, Dong Q, et al: Molecular properties of $\mathrm{CD}_{133^{+}}$glioblastoma stem cells derived from treatment-refractory recurrent brain tumors. J Neurooncol 94: 1-19, 2009.

10. Spencer DD, Robbins RJ, Naftolin F, et al: Unilateral transplantation of human fetal mesencephalic tissue into the caudate nucleus of patients with Parkinson's disease. N Engl J Med 327: 1541-1548, 1992

11. Freed CR, Breeze RE, Rosenberg NL, et al: Survival of implanted fetal dopamine cells and neurologic improvement 12 to 46 months after transplantation for Parkinson's disease. N Engl J Med 327: 1549-1555, 1992.

12. Imitola J, Raddassi K, Park KI, et al: Directed migration of neural stem cells to sites of CNS injury by the stromal cell-derived factor 1alpha/CXC chemokine receptor 4 pathway. Proc Natl Acad Sci USA 101: 18117-18122, 2004

13. Armstrong RJ and Svendsen CN: Neural stem cells: from cell biology to cell replacement. Cell Transplant 9: 139-152, 2000.

14. Carbajal KS, Schaumburg C, Strieter R, Kane J and Lane TE: Migration of engrafted neural stem cells is mediated by CXCL12 signaling through CXCR4 in a viral model of multiple sclerosis. Proc Natl Acad Sci USA 107: 11068-11073, 2010.

15. Aboody KS, Brown A, Rainov NG, et al: Neural stem cells display extensive tropism for pathology in adult brain: evidence from intracranial gliomas. Proc Natl Acad Sci USA 97: 12846-12851, 2000.

16. Benedetti S, Pirola B, Pollo B, et al: Gene therapy of experimental brain tumors using neural progenitor cells. Nat Med 6: 447-450, 2000

17. Herrlinger U, Woiciechowski C, Sena-Esteves M, et al: Neural precursor cells for delivery of replication-conditional HSV-1 vectors to intracerebral gliomas. Mol Ther 1: 347-357, 2000.

18. Staflin K, Honeth G, Kalliomaki S, Kjellman C, Edvardsen K and Lindvall M: Neural progenitor cell lines inhibit rat tumor growth in vivo. Cancer Res 64: 5347-5354, 2004.

19. Glass R, Synowitz M,Kronenberg G, et al: Glioblastoma-induced attraction of endogenous neural precursor cells is associated with improved survival. J Neurosci 25: 2637-2646, 2005.

20. Yip S, Sabetrasekh R, Sidman RL and Snyder EY: Neural stem cells as novel cancer therapeutic vehicles. Eur J Cancer 42: 1298-1308, 2006.

21. Kim SU: Neural stem cell-based gene therapy for brain tumors. Stem Cell Rev 7: 130-140, 2011.

22. Spencer D: Fighting brain tumors while protecting the brain: the stem cell story. Neurology 76: e69-e70, 2011.

23. Yu JJ, Sun X, Yuan X, Lee JW, Snyder EY and Yu JS: Immunomodulatory neural stem cells for brain tumour therapy. Expert Opin Biol Ther 6: 1255-1262, 2006.

24. Ahmed AU, Alexiades NG and Lesniak MS: The use of neural stem cells in cancer gene therapy: predicting the path to the clinic. Curr Opin Mol Ther 12: 546-552, 2010.

25. Aboody KS, Najbauer J and Danks MK: Stem and progenitor cell-mediated tumor selective gene therapy. Gene Ther 15: 739-752, 2008

26. Zhang SJ, Ye F, Xie RF, et al: Comparative study on the stem cell phenotypes of C6 cells under different culture conditions. Chin Med J 124: 3118-3126, 2011.

27. Campos B, Wan F, Farhadi M, et al: Differentiation therapy exerts antitumor effects on stem-like glioma cells. Clin Cancer Res 16: 2715-2728, 2010. 
28. Laks DR, Masterman-Smith M, Visnyei K, et al: Neurosphere formation is an independent predictor of clinical outcome in malignant glioma. Stem Cells 27: 980-987, 2009.

29. Zhang S, Luo X, Wan F and Lei T: The roles of hypoxia-inducible factors in regulating neural stem cells migration to glioma stem cells and determinating their fates. Neurochem Res 37: 2659-2666, 2012.

30. Busletta C, Novo E, Valfre DBL, et al: Dissection of the biphasic nature of hypoxia-induced motogenic action in bone marrowderived human mesenchymal stem cells. Stem Cells 29: 952-963, 2011.

31. Kendall SE, Najbauer J, Johnston HF, et al: Neural stem cell targeting of glioma is dependent on phosphoinositide 3-kinase signaling. Stem Cells 26: 1575-1586, 2008.

32. Schmidt NO, Przylecki W, Yang W, et al: Brain tumor tropism of transplanted human neural stem cells is induced by vascular endothelial growth factor. Neoplasia 7: 623-629, 2005.

33. Mercapide J, Rappa G, Anzanello F, King J, Fodstad O and Lorico A: Primary gene-engineered neural stem/progenitor cells demonstrate tumor-selective migration and antitumor effects in glioma. Int J Cancer 126: 1206-1215, 2010.

34. Pollard SM, Conti L, Sun Y, Goffredo D and Smith A: Adherent neural stem (NS) cells from fetal and adult forebrain. Cereb Cortex 16 (Suppl 1): i112-i120, 2006.

35. Sun Y, Pollard S, Conti L, et al: Long-term tripotent differentiation capacity of human neural stem (NS) cells in adherent culture. Mol Cell Neurosci 38: 245-258, 2008.

36. Pollard SM, Yoshikawa K, Clarke ID, et al: Glioma stem cell lines expanded in adherent culture have tumor-specific phenotypes and are suitable for chemical and genetic screens. Cell Stem Cell 4: 568-580, 2009.
37. Xu Q, Wang S, Jiang X, et al: Hypoxia-induced astrocytes promote the migration of neural progenitor cells via vascular endothelial factor, stem cell factor, stromal-derived factor-1alpha and monocyte chemoattractant protein-1 upregulation in vitro. Clin Exp Pharmacol Physiol 34: 624-631, 2007.

38. Zhao D, Najbauer J, Garcia E, et al: Neural stem cell tropism to glioma: critical role of tumor hypoxia. Mol Cancer Res 6: 1819-1829, 2008.

39. Pistollato F, Abbadi S, Rampazzo E, et al: Intratumoral hypoxic gradient drives stem cells distribution and MGMT expression in glioblastoma. Stem Cells 28: 851-862, 2010.

40. Chirasani SR, Sternjak A, Wend P, et al: Bone morphogenetic protein-7 release from endogenous neural precursor cells suppresses the tumourigenicity of stem-like glioblastoma cells. Brain 133: 1961-1972, 2010.

41. Kim SK, Kim SU, Park IH, et al: Human neural stem cells target experimental intracranial medulloblastoma and deliver a therapeutic gene leading to tumor regression. Clin Cancer Res 12: 5550-5556, 2006

42. van Eekelen M, Sasportas LS, Kasmieh R, et al: Human stem cells expressing novel TSP-1 variant have anti-angiogenic effect on brain tumors. Oncogene 29: 3185-3195, 2010.

43. Ehtesham M, Kabos P, Gutierrez MA, et al: Induction of glioblastoma apoptosis using neural stem cell-mediated delivery of tumor necrosis factor-related apoptosis-inducing ligand. Cancer Res 62: 7170-7174, 2002. 